Journal for ImmunoTherapy of Cancer

\title{
Microwave ablation induces Th1-type immune response with activation of ICOS pathway in early-stage breast cancer
}

\author{
Wenbin Zhou (D) , ${ }^{1,2}$ Muxin Yu, ${ }^{1,2}$ Hong Pan, ${ }^{1,2}$ Wen Qiu, ${ }^{3}$ Hui Wang, ${ }^{1}$ Mengjia Qian, ${ }^{1}$ \\ Nan Che, ${ }^{4}$ Kai Zhang, ${ }^{5}$ Xinrui Mao, ${ }^{1}$ Li Li, ${ }^{6}$ Ruoxi Wang, ${ }^{1}$ Hui Xie, ${ }^{1,2}$ Lijun Ling, ${ }^{1,2}$ \\ Yi Zhao, ${ }^{1,2}$ Xiaoan Liu, ${ }^{1,2}$ Cong Wang, ${ }^{7}$ Qiang Ding, ${ }^{1,2}$ Shui Wang ${ }^{1,2}$
}

To cite: Zhou W, Yu M, Pan H, et al. Microwave ablation induces Th1-type immune response with activation of ICOS pathway in early-stage breast cancer. Journal for ImmunoTherapy of Cancer 2021;9:e002343. doi:10.1136/ jitc-2021-002343

WZ, MY, HP and WQ contributed equally.

Accepted 09 March 2021

\section{Check for updates}

(c) Author(s) (or their employer(s)) 2021. Re-use permitted under CC BY-NC. No commercial re-use. See rights and permissions. Published by BMJ.

For numbered affiliations see end of article.

\section{Correspondence to} Dr Wenbin Zhou, Department of Breast Surgery, The First Affiliated Hospital with Nanjing Medical University, Nanjing,

China;

zhouwenbin@njmu.edu.cn

Professor Shui Wang, Department of Breast Surgery, The First Affiliated Hospital with Nanjing Medical University,

Nanjing, China;

shwang@njmu.edu.cn

\section{ABSTRACT}

Background Despite great advances in the treatment of breast cancer, innovative approaches are still needed to reduce metastasis. As a minimally invasive local therapy (not standard therapy for breast cancer), microwave ablation (MWA) has been attempted to treat breast cancer, but the local effect and immune response induced by MWA have seldom been reported.

Methods The clinical study was performed to determine the complete ablation rate of MWA for early-stage breast cancer. Secondary endpoints included safety and antitumor immune response. 35 subjects from this clinical study were enrolled in the current report, and the local effect was determined by pathological examinations or follow-up. To investigate MWA-induced immune response, patients treated with surgery $(n=13)$ were enrolled as control, and blood samples were collected before and after MWA or surgery. The immune cell populations, serum cytokines, secretory immune checkpoint molecules, and T-cell receptor sequencing were analyzed.

Results Of 35 enrolled patients, 32 (91.4\%) showed complete ablation. Compared with surgery, MWA induced significantly increased levels of inducible co-stimulator (ICOS)+ activated CD4+ T cells and serum interferon gamma, indicating a shift in the Th1/Th2 balance toward Th1. The activated ICOS pathway was involved in the MWA-induced adaptive immune response. T-cell receptor sequencing revealed MWA of primary tumor activated T lymphocytes expansion and recognized some cancerspecific antigens. Moreover, CD4+ effector memory T-cell response was induced by MWA, and the immune response still existed after surgical resection of the ablated tumor. Conclusions MWA may not only be a promising local therapy but also a trigger of antitumor immunity for breast cancer, opening new avenues for the treatment of breast cancer. Combinatorial strategy using additional agents which boost MWA-induced immune response could be considered as potential treatment for clinical study for early breast cancer therapy.

\section{BACKGROUND}

Breast cancer is a significant threat to women's health. ${ }^{1}$ Although the survival has been significantly improved due to advances in early detection and therapy, some patients still suffer from metastatic breast cancer even after systemic therapies, especially for triplenegative breast cancer (TNBC). Therefore, innovative approaches for breast cancer therapy are still needed to reduce metastasis and relapse. ${ }^{23}$ Immunotherapy is an attractive treatment strategy especially for highly immunogenic cancers. ${ }^{45}$ Immune checkpoint antagonists specific for CTLA-4, PD-1 and PD-L1 have been tested in advanced breast cancer. ${ }^{356}$ However, the objective response rate is relatively low in several subtypes of relatively 'cold' tumors, ${ }^{2}{ }^{6}$ which may be partially explained by the low antigenicity of these subtypes of breast cancer. ${ }^{78}$ Innovative approaches which can induce immune responses against tumor-associated antigens may be a promising research direction. ${ }^{8-11}$

Surgery is the standard local treatment for early-stage breast cancer. However, systemic inflammation and surgery-associated immunosuppression during the postoperative period are reported in several studies. ${ }^{12-14}$ Moreover, surgical stress induces a shift in the Th1/Th2 balance toward Th2, ${ }^{14}$ so less invasive surgical procedures are recommended. Due to the advantages over surgery, minimally invasive thermal therapies have been attempted to treat breast cancer and other solid tumors. ${ }^{915}$ In situ tumor ablation can create an antigen source for the generation of antitumor immunity. ${ }^{16}$ As an effective local treatment for solid tumors, ablation-related immuno-modulation has been reported in preclinical studies. ${ }^{9} 10$ 17-20 Importantly, increased peripheral T-cell population and T-cell infiltration after thermal ablation have been observed mainly in liver tumors. ${ }^{21} 22$ Moreover, NK cell response has only been reported after radiofrequency ablation of 
liver tumors in one study. ${ }^{23}$ Peripheral elevated levels of inducible co-stimulator+ (ICOS+, a member of the CD28 costimulatory family, as a surrogate of T-cell activation) activated $\mathrm{T}$ cells were observed in six patients treated with cryoablation combined with ipilimumab in a previous study. ${ }^{8}$ Based on several advantages, microwave ablation (MWA) has been attempted in the treatment of breast cancer in limited feasibility studies ${ }^{24}{ }^{25}$ about ablation of small tumors followed by immediately resection. To the best of our knowledge, the immune response induced by ablation for breast cancer has seldom been reported.

This clinical study was performed to determine the complete ablation rate of MWA for breast cancer confirmed by delayed surgery or follow-up. Secondary and exploratory endpoints included safety and antitumor immune response. Herein, we found MWA was a promising local therapy for breast cancer with a high complete ablation rate. Moreover, MWA induced Th1-type immune response in women with early-stage breast cancer. Importantly, the ICOS pathway was activated in MWA-induced immune response. Furthermore, CD4+ T-cell memory formation was initiated by MWA. As a minimally invasive local therapy, MWA may not only be a promising local therapy but also a trigger of antitumor immunity for breast cancer. Therefore, MWA may be a promising alternative to surgery for the local therapy of breast cancer, although future studies are recommended to confirm our results.

\section{METHODS}

\section{Study design}

A single-arm, multicenter clinical study was performed to determine the local effect of MWA in the treatment of early-stage breast cancer. Secondary and exploratory endpoints included safety and antitumor immune response.

From July 2016 to June 2019, 35 subjects who underwent MWA successfully were enrolled in this study. From August 2018 to December 2018, 13 consecutive patients diagnosed with invasive breast cancer with similar clinical stages to those in the MWA group, who underwent standard surgery, were included as controls for the analysis of immune response (figure 1B). The main exploratory objective was to determine MWA-induced immune response 1 week after ablation, so the peripheral blood was examined before and 1 week after MWA or surgery (figure 1A). The blood was also examined 4 weeks after MWA in selected cases to determine whether immune responses still exist after surgical resection of the ablated tumor.

\section{Patient enrollment for MWA}

The patients diagnosed with invasive breast cancer by core needle biopsy, with a maximal diameter of $\leq 3 \mathrm{~cm}$ proved by ultrasound, were enrolled in the single-arm, multicenter clinical study. Before any treatment, the status of hormone receptor, human epidermal growth factor
A
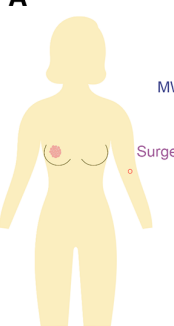
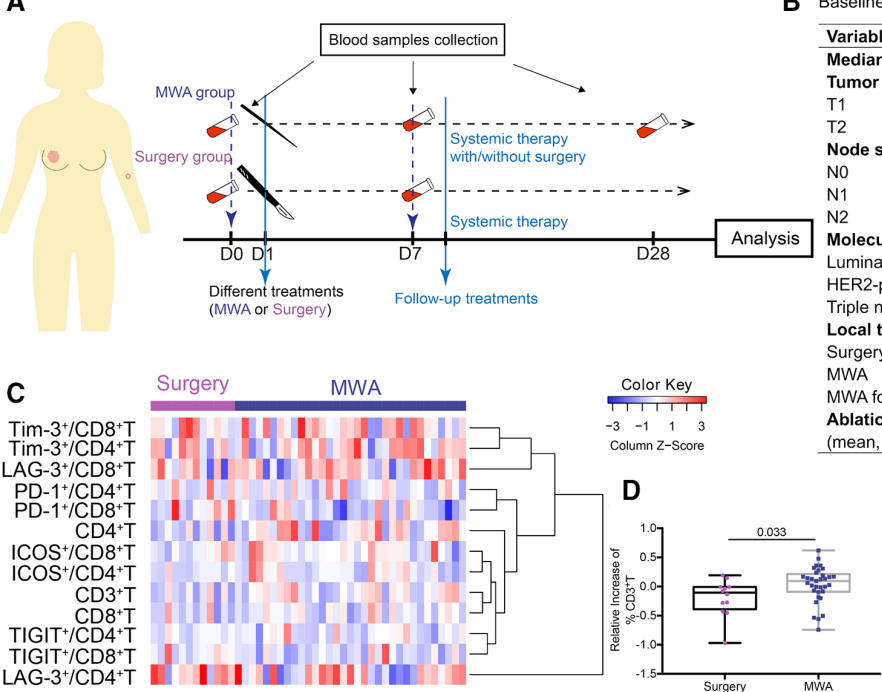

\begin{tabular}{|c|c|c|}
\hline Variables & Surgery (13) & Microwave ablation (35) \\
\hline Median age, $y$ & $46(32-64)$ & $59(38-87)$ \\
\hline \multicolumn{3}{|l|}{ Tumor size } \\
\hline $\mathrm{T} 1$ & $6(46.2 \%)$ & $20(57.1 \%)$ \\
\hline T2 & $7(53.8 \%)$ & $15(42.9 \%)$ \\
\hline \multicolumn{3}{|l|}{ Node status } \\
\hline No & $4(30.8 \%)$ & $29(82.9 \%)$ \\
\hline N1 & $5(38.5 \%)$ & $4(11.4 \%)$ \\
\hline N2 & $4(30.8 \%)$ & $2(5.7 \%)$ \\
\hline \multicolumn{3}{|l|}{ Molecular subtype } \\
\hline Luminal-HER2-negative & $8(61.5 \%)$ & $20(57.1 \%)$ \\
\hline HER2-positive & $2(15.4 \%)$ & $7(20.0 \%)$ \\
\hline Triple negative & $3(23.1 \%)$ & $8(22.9 \%)$ \\
\hline \multicolumn{3}{|l|}{ Local treatment } \\
\hline Surgery & $13(100 \%)$ & $0(0 \%)$ \\
\hline MWA & $0(0 \%)$ & $15(42.9 \%)$ \\
\hline MWA followed by surgery & $0(0 \%)$ & $20(57.1 \%)$ \\
\hline Ablation time, minutes & 1 & $\begin{array}{l}2.5 \\
(2-5)\end{array}$ \\
\hline
\end{tabular}

$$
\text { E }
$$

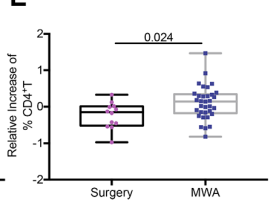

\section{$\mathbf{F}$}

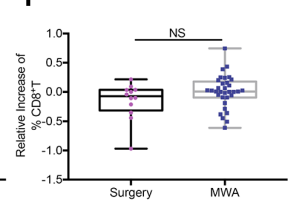

G
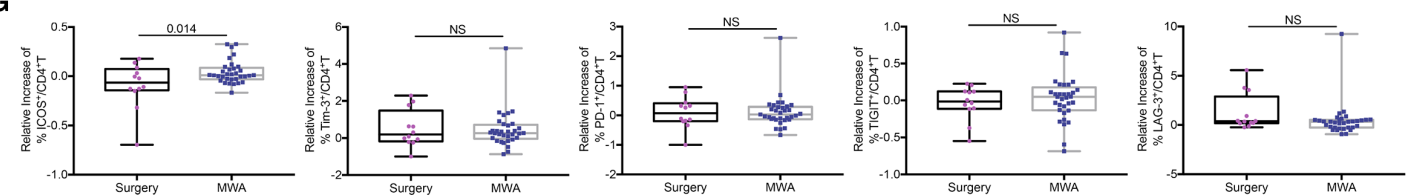

Figure 1 Phenotypical characterization of peripheral T cells in patients treated with MWA. (A) Schematic illustrating the study design. (B) Basic characteristics of enrolled patients.(C) Heat map of the changes in peripheral T cells, CD4+, and CD8+ Tcell subsets in the MWA group $(n=33)$ and the surgery group $(n=12)$. The increased frequencies of peripheral T cells (D), CD4+ $(E)$, but not CD8+ (F) T cells in patients treated with MWA were compared with those in surgery. (G) Scatter plots showing the changes in the activated (ICOS) and exhausted (LAG-3, TIGIT, TIM-3 and PD-1) CD4+ T cells. Data are presented as mean \pm SD. HER2, human epidermal growth factor receptor 2; ICOS, inducible co-stimulator; MWA, microwave ablation; NS, not significant. 
receptor 2 (HER2), and Ki67 was evaluated. Only a single tumor, without an extensive intraductal component and infiltration to the skin or pectoralis major muscle, was included.

\section{MWA and other treatments}

In the MWA group, all enrolled patients underwent MWA successfully. If they declined or were unsuitable for surgery due to medical conditions, surgery was not performed. Otherwise, prescheduled surgery was performed over 1 week after MWA. Subsequently, other systemic therapies were recommended according to the guidelines.

MWA was performed under local anesthesia according to the guidelines in our center. ${ }^{24}{ }^{25}$ The microwave irradiation frequency of the system (Nanjing Yigao Microwave Electric Institute, Nanjing, China) was $2450 \mathrm{MHz}$ with an output power set at $40 \mathrm{~W}$. According to our previous experiences, ${ }^{25}{ }^{26}$ MWA for at least 2 min was recommended to achieve complete ablation for tumors larger than $1 \mathrm{~cm}$, and at least $1 \mathrm{~min}$ for tumors smaller than $1 \mathrm{~cm}$. Two to five minutes were needed for MWA in this study. Complete ablation was defined as the tumor disappearing completely on ultrasound. Any complication related to MWA was assessed based on pathology or follow-up imaging.

\section{Clinical assessment of local effect for MWA}

For patients who underwent MWA without local surgery, breast MRI and contrast-enhanced ultrasound was recommended 1 month after MWA and then every year during follow-up. Breast ultrasound was recommended half a year, and mammography was performed every year after MWA. Complete ablation was defined as no focal enhancement within or at the periphery of the tumor confirmed by contrast-enhanced imagines. ${ }^{27}$ When suspected local recurrence was observed, biopsy or local surgery was recommended. For patients who underwent MWA followed by surgery, complete ablation was defined as no viable tumor cells determined by pathological examinations. ${ }^{25}$

More details about methods of this study are available in online supplemental materials.

\section{Statistical analysis}

Numerical data are reported as mean \pm SD. The percentage changes from baseline (relative increase) of T-cell markers were calculated. The difference in the changes between the MWA and surgery groups was determined by the parametric test. The difference before and after MWA or surgery was assessed by Student's paired t-test. A $p$ valueof $<0.05$ was considered statistically significant.

Unsupervised clustering of $\mathrm{T}$ cells in breast cancer samples of patients with MWA or surgery was performed by FlowSOM packages in R/Bioconductor (source repository: git clone https://git.bioconductor.org/packages/FlowSOM), which is an algorithm that uses selforganizing maps (SOMs) for automated clustering and dimensionality reduction. Briefly, five steps are provided in this package: first, the data are read and preprocessed. Then, SOMs are built based on the data contained in the FlowSOM object, and the distance matrix is calculated by a minimal spanning tree neighborhood. The fourth step is to perform a metaclustering of the data. Finally, the FlowSOM grid or tree is plotted, in which each node is represented by a star chart indicating median marker values.

\section{RESULTS \\ Characteristics of the study population and local effect of MWA}

Thirty-five subjects from this trial, in whom MWA was successfully performed, were enrolled in the present study. The baseline clinical characteristics are shown in figure 1B (detailed in online supplemental table 1). All patients were diagnosed with invasive breast cancer $3.0 \mathrm{~cm}$ or less in diameter. Of these 35 patients, 15 patients, who declined or were not candidates for surgery due to medical conditions, underwent MWA without local surgery, and 20 underwent MWA followed by prescheduled surgery 1 week later. Of these 20 cases, unexpected intraductal carcinoma was found beyond the targeted tumor in three cases, defined as no complete ablation. Of the 15 patients treated with MWA (detailed therapies in online supplemental table 2), no local recurrence was observed with a median follow-up of 36 months (range 13-47 months). Of all these 35 cases, 32 (91.4\%) showed complete ablation confirmed by follow-up or pathological examinations. All $(100 \%)$ showed local swelling at the treatment site about 2-3 days after ablation which then disappeared in 1 week (detailed complications in online supplemental table 3).

\section{Phenotypical characterization of T cells in peripheral blood}

The peripheral blood was obtained before and 1 week after MWA, not influenced by following surgery or systemic therapy (figure 1A). Thirteen other patients with early-stage breast cancer, who underwent surgery only, were enrolled as control (figure 1B), and the peripheral blood was examined before and 1 week after surgery (figure 1A).

The changes in peripheral $\mathrm{T}$ cells, CD4+, and CD8+ T-cell subsets were compared between the two groups (figure 1C). The increased percentage of peripheral $\mathrm{T}$ cells 1 week after MWA ( $n=33$ ) was significantly higher than that in the surgery group $(n=12) \quad(3.37 \% \pm 30.31 \%$ vs $-19.42 \% \pm 31.82 \%, \mathrm{p}=0.033$; figure $1 \mathrm{D})$. Interestingly, the increased percentage of peripheral CD4+ T cells was significantly larger than that in the surgery group $(10.68 \% \pm 46.04 \%$ vs $-23.81 \% \pm 35.91 \%, \mathrm{p}=0.024$; figure $1 \mathrm{E}$ ), but no significant difference was observed for CD8+ T cells $(0.82 \% \pm 27.84 \%$ vs $-15.44 \% \pm 31.59 \%$, $\mathrm{p}=0.102$; figure $1 \mathrm{~F}$ ). When ICOS expression was evaluated as a surrogate of T-cell activation, a significant increase in the proportion of activated T cells after MWA was observed in the CD4+ subset $(4.04 \% \pm 11.69 \%$ vs $-9.37 \% \pm 23.48 \%$, $\mathrm{p}=0.014$; figure $1 \mathrm{G})$ but not in the CD8+ subset $(\mathrm{p}=0.475$, 
online supplemental figure $1 \mathrm{~A})$ compared with that in the surgery group. When LAG-3, TIGIT, TIM-3, and PD-1 were evaluated as exhausted phenotype, there was no significant change between the two groups in the proportion of exhausted T cells in either CD4+ (figure 1G) or CD8+ subset (online supplemental figure 1A).

The peripheral frequencies of all T cells, CD4+, or CD8+ $\mathrm{T}$ cells after MWA were not significantly higher than those before MWA (online supplemental figure 1B). However, the frequencies of ICOS+CD4+ and ICOS+CD8+ T cells seemed to increase after MWA (online supplemental figure 1C).

\section{MWA-induced upregulation of Th1-associated cytokines}

Peripheral Th1 and Th2 cytokines were measured before and 1 week after therapy (figure 2A). Th1 cytokine interferon gamma (IFN- $\gamma$ ) but not interleukin (IL)-2, IL-12 or IL-18 significantly increased after MWA $(\mathrm{n}=32)$ compared with surgery $(n=13)$ (figure $2 B)$. In contrast, the differences of Th2 cytokines IL-4 and IL-10 between surgery and MWA groups were not significant (figure 2A). In addition, proinflammatory cytokine IL-6 exhibited a 2.76-fold increase after surgery, but only increased a little after MWA, with a significant difference between the two groups ( $p=0.002$, figure $2 B$ ). No significant difference in

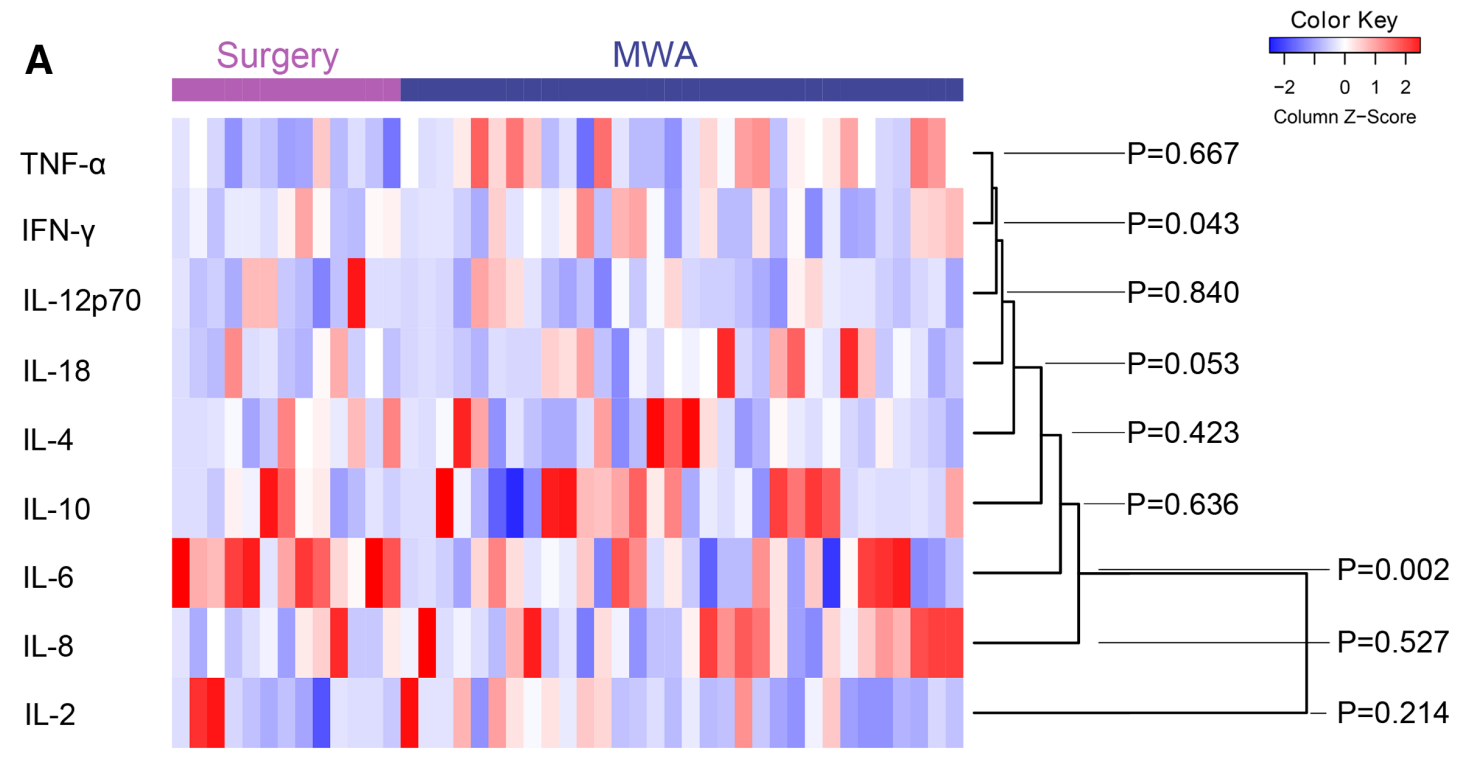

B Th1-type cytokine Proinflammatory cytokine

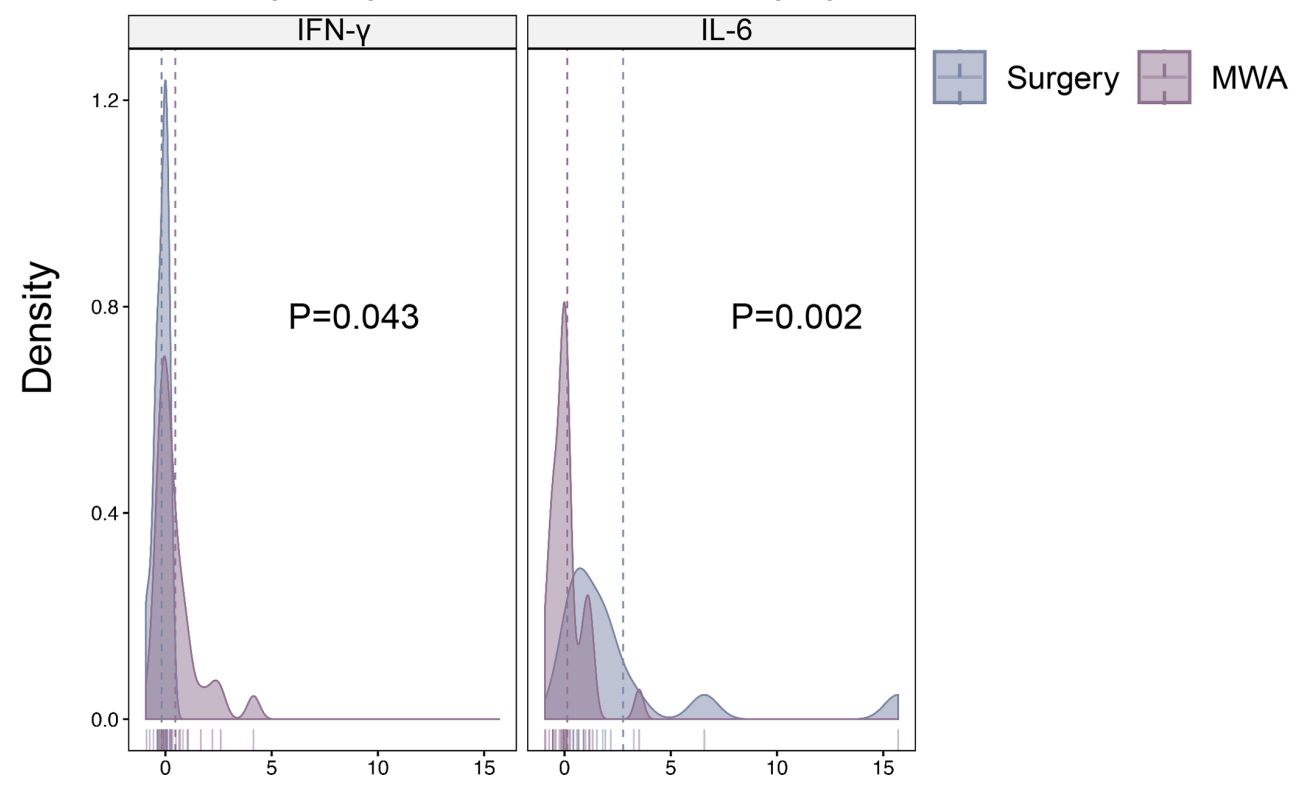

Figure 2 Changes in peripheral cytokines induced by MWA in comparison to surgery. (A) Heat map of the changes in peripheral cytokines in the MWA group $(n=32)$ and the surgery group $(n=13)$. (B) Scatter plots showing the changes in peripheral Th1-associated cytokine IFN- $\gamma$ and proinflammatory cytokine IL- 6 between the two groups. Data are presented as mean \pm SD. IFN- $\gamma$, interferon gamma; IL, interleukin; MWA, microwave ablation; TNF- $\alpha$, tumor necrosis factor alpha. 

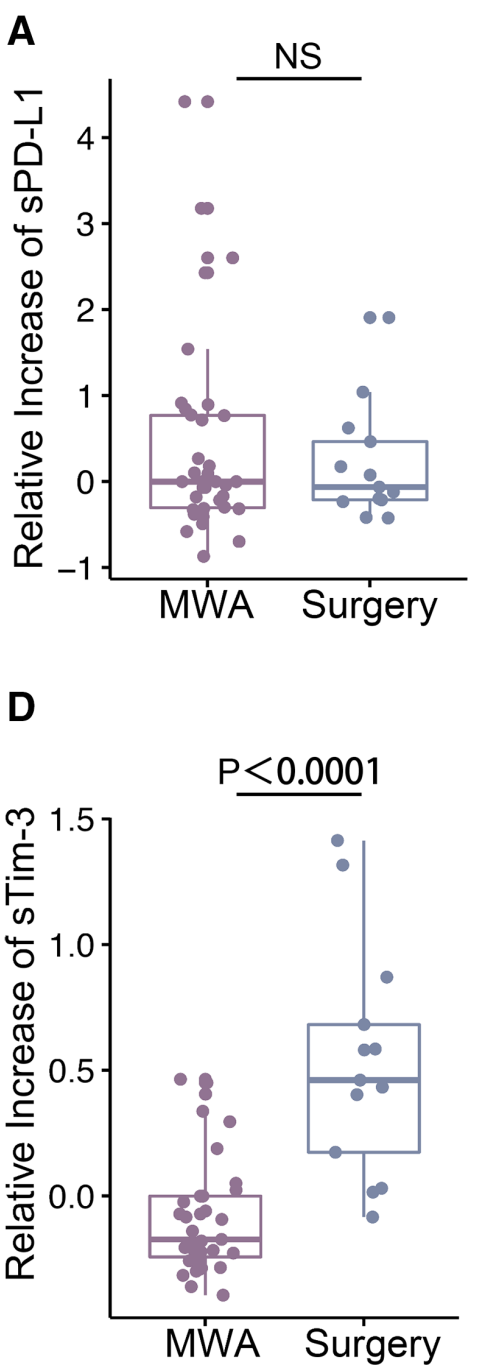
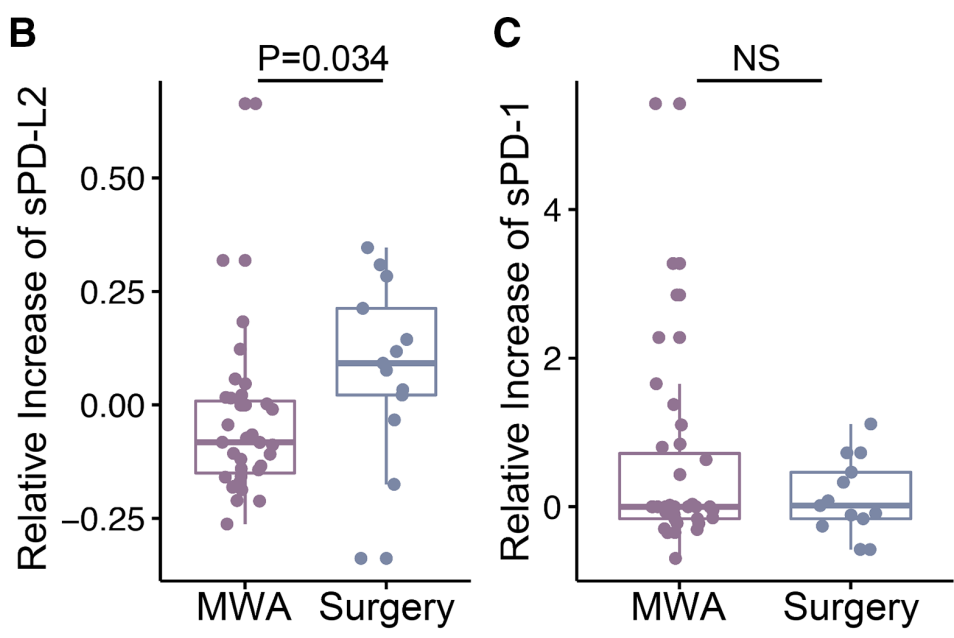

E

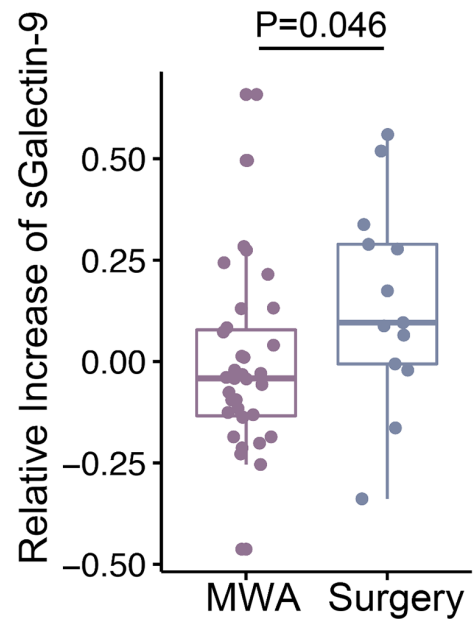

$\mathbf{F}$

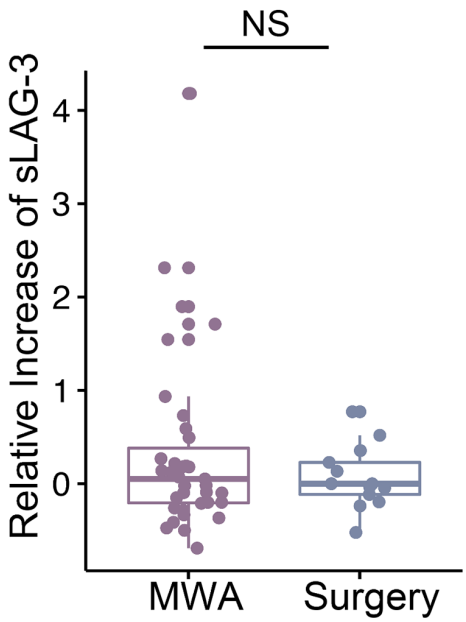

Figure 3 Changes in secretory immune checkpoint molecules in the blood. The changes in sPD-L1 (A), sPD-1 (C) and sLAG-3 $(F)$ in the MWA group $(n=35)$ and surgery group $(n=13)$ were not significantly different. sPD-L2 (B), sTIM-3 (D) and sGalectin-9 (E) decreased after MWA, with significant differences from that in surgery group. Data are presented as mean \pm SD. MWA, microwave ablation; NS, not significant.

the changes in IL-8 and tumor necrosis factor alpha was observed between the two groups.

\section{MWA-induced secretory immune checkpoint molecules different from surgery}

In vivo anticancer immune response may be counterbalanced by the number of immune checkpoints. Soluble immune checkpoint molecules in the blood may reflect host immunity and indicate the effect of therapeutic strategies for patients with cancer. ${ }^{28-30}$ To further determine the immune response induced by MWA, soluble PD-L1 (sPD-L1), PD-L2 (sPD-L2), PD-1 (sPD-1), TIM-3 (sTIM3), LAG-3 (sLAG-3) and galectin-9 (sGalectin-9) were examined in peripheral blood before and 1 week after MWA (figure 3A-F).

Among 13 surgery cases, levels of all soluble immune checkpoint molecules listed earlier increased after surgery. On the other hand, in all 35 MWA cases, levels of sPD-L1, sPD-1 and sLAG-3 also slightly increased after MWA, which were similar to those in the surgery group (figure 3A,C and F). Interestingly, levels of sPD-L2, sTIM-3 and sGalectin-9 decreased after MWA, with significant differences from those in surgery group (figure 3B,D and E).

\section{Upregulation of memory T cells induced by MWA of breast cancer}

In response to antigenic challenge, naïve $\mathrm{T}$ cells proliferate and differentiate. Then over $95 \%$ of these cells die later after antigenic clearance, leaving behind a small pool of long-lived memory cells. Memory T cells turn into effector T cells on encountering the antigen and provide effective secondary immunoresponses. ${ }^{31}{ }^{32}$ The changes in peripheral memory T-cell subsets were examined for 31 patients in the MWA group and 12 patients in the surgery group (figure 4).

Major functional subsets of $\mathrm{T}$ cells are delineated by the expression of the cell surface CD45 isoform CD45RA and chemokine receptor CCR7, ${ }^{33}$ including naïve $\left(\mathrm{CD}_{4} 5 \mathrm{RA}^{+} \mathrm{CCR} 7^{+}\right)$, central memory 
A
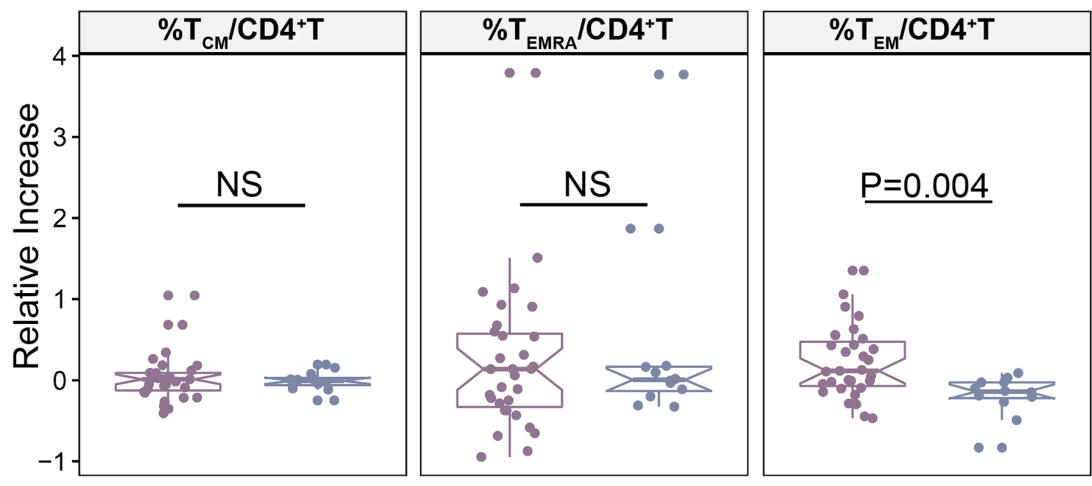

MWA

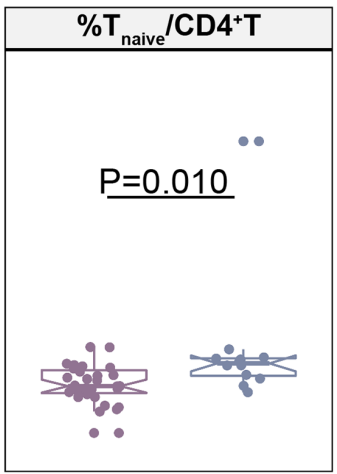

B
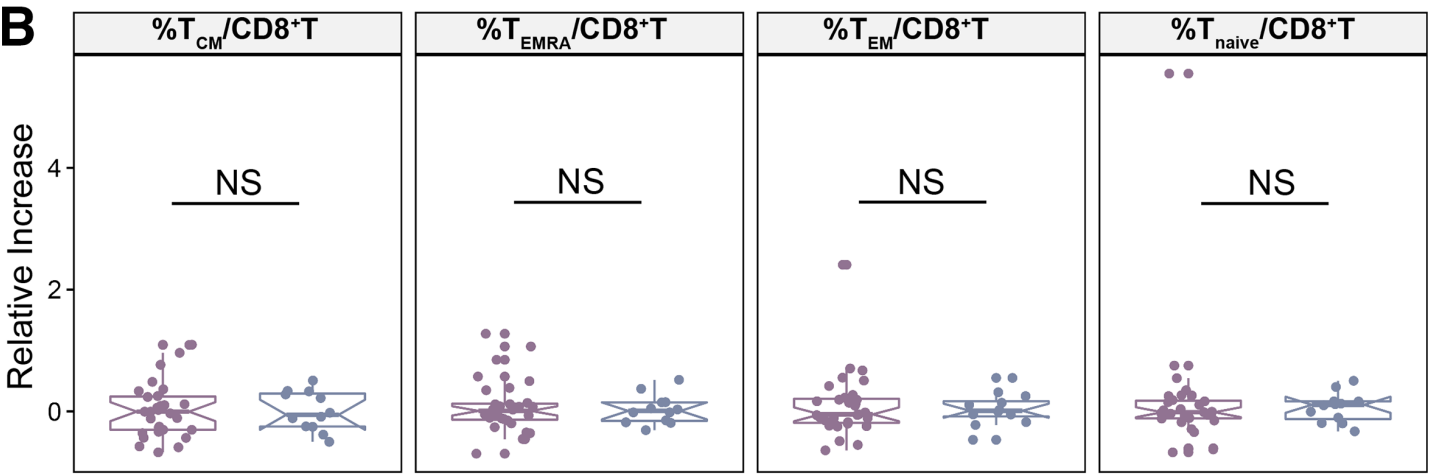

C

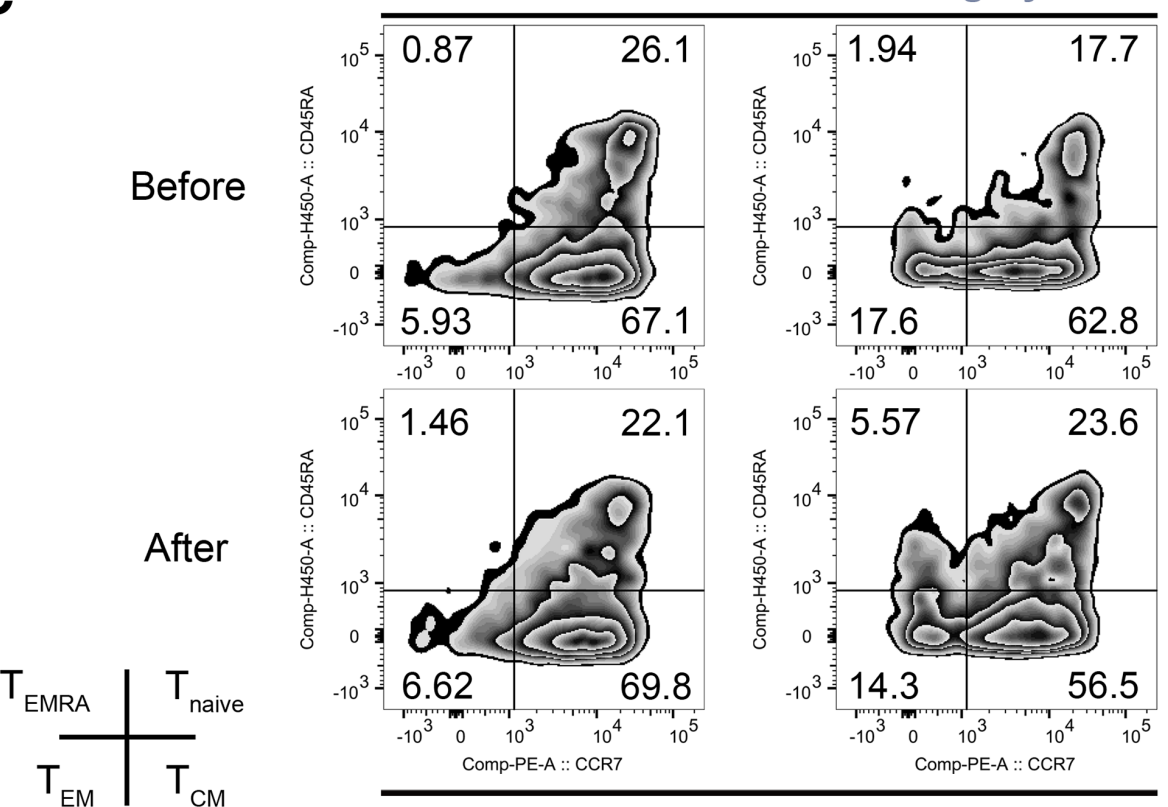

Gate on $\mathrm{CD}^{+} \mathrm{T}$

Figure 4 Changes in peripheral memory T-cell subsets for 31 patients in the MWA group and 12 patients in the surgery group. The changes in CD4+ (A) and CD8+ (B) $T_{\text {naive }}, T_{C M}, T_{E M}$, and $T_{E M R A}$ are shown. (C) Representative flow cytometric plots showing $C D 4+T_{\text {naive }}, T_{C M}, T_{E M}$, and $T_{E M P A}$ before and after MWA or surgery. Data are presented as mean $\pm S D$. MWA, microwave ablation; $N S$, not significant; $T_{C M}$, central memory $T$ cell; $T_{E M}$, effector memory $T$ cell; $T_{E M R A}$, terminal effector $T$ cell; $T_{\text {naive, }}$, naïve $T$ cell.

$\mathrm{T}$ cell $\left(\mathrm{T}_{\mathrm{CM}}, \mathrm{CD} 45 \mathrm{RA}^{-} \mathrm{CCR} 7^{+}\right)$, effector memory $\mathrm{T}$ cell $\left(\mathrm{T}_{\mathrm{EM}}, \mathrm{CD} 45 \mathrm{RA}^{-} \mathrm{CCR} 7^{-}\right)$, and terminal effector $\mathrm{T}$ cell $\left(\mathrm{CD} 45 \mathrm{RA}^{+} \mathrm{CCR} 7^{-}\right)$cell subsets. The percentage of naive CD4+ T cells decreased after MWA and increased after surgery, with significant differences $(-9.37 \% \pm 23.00 \%$ vs $33.55 \% \pm 82.21, \mathrm{p}=0.010$; figure $4 \mathrm{~A}, \mathrm{C}) . \mathrm{CD} 4+\mathrm{T}_{\mathrm{FM}}$ significantly increased after MWA (online supplemental figure $2, p=0.035)$, and the increased level of $\mathrm{CD} 4+\mathrm{T}_{\mathrm{EM}}$ in 
MWA group was significantly higher than that in surgery group $(22.12 \% \pm 43.52 \%$ vs $-19.03 \% \pm 25.25, \quad \mathrm{p}=0.004$; figure $4 \mathrm{~A}, \mathrm{C}) . \mathrm{CD} 4+\mathrm{T}_{\mathrm{CM}}$ and $\mathrm{T}_{\mathrm{EMRA}}$ as well as CD8+ T-cell subsets were similar in both groups (figure 4A,B).

\section{Peripheral clonal T-cell expansions after MWA}

In situ tumor ablation may create a tumor antigen source for the generation of antitumor immunity. ${ }^{16}$ To determine whether MWA of breast cancer can induce tumor antigen release and peripheral clonal T-cell expansions, we used multiplex PCR to amplify the T-cell receptor $\beta$ (TCR $\beta$ ) chain and then investigated the sequence of TCR $\beta$ chain using ultradeep sequencing on pre-MWA and post-MWA. Paired peripheral blood mononuclear cell (PBMC) samples were obtained from five patients (online supplemental methods and table 1). Through cDNA sequencing of the TCR $\beta$ chain, we detected TCR $\beta$ sequence reads of $7095139 \pm 2768841$ (range from 3052282 to 10475722 (online supplemental table 4), distributed $503 \quad 814 \pm 132260$ TCR $\beta$ complementarity determining region 3 (CDR3) clonotypes in PBMC specimens. We calculated the proportions of abundant CDR3 clonotypes (defined as the clonotypes with the frequency of $0.1 \%$ or higher), and found that the sum of the abundant CDR3 clonotypes was significantly increased after MWA ( $20.56 \% \pm 9.07$ vs $25.90 \% \pm 7.83$, paired t test $\mathrm{p}=0.013$, figure 5). To further illustrate the TCR $\beta$ CDR3 clonotypes enriched after MWA, the top 20 abundant TCR $\beta$ CDR3 clonotypes in pretreatment and post-treatment specimens were compared, and most of the enriched TCR $\beta$ CDR3 clonotypes in post-MWA samples were absent or rarely present in pre-MWA samples (figure 5).

\section{Activated ICOS pathway in MWA-induced activated CD4+ T cells}

To understand T-cell subpopulations in more granular detail, unsupervised clustering analysis was performed (figure 6A and online supplemental figure 3). Before any treatment, clustering around twocell populations were observed: $\mathrm{CD} 3+\mathrm{CD} 4+$ cells with moderate PD-1 and ICOS expression (online supplemental figure $3 \mathrm{~A}$ ), and CD3 +CD8+ cells with moderate to high PD-1 expression (figure 6A and online supplemental figure 3A). After MWA, CD3+CD4+ICOS+ cell population was obviously increased (figure 6A). Moreover, CD3+CD8+ICOS+cell population was found in several cases after MWA (figure 6A). From the cluster maps, the expression of CD4 and ICOS mainly increased after MWA but not surgery.

It has been reported that ICOS is upregulated following T-cell activation ${ }^{34}$ and plays an important role in T-cell memory regulation, especially for CD4+ T cells. ${ }^{35}{ }^{36} \mathrm{We}$ found that CD4+ T cells were activated (ICOS+), and CD4+ $\mathrm{T}_{\mathrm{EM}}$ also increased after MWA in this study. The ICOS pathway may be activated by MWA of breast cancer. To further confirm this observation, reverse transcription PCR of peripheral T cells was performed in six MWA cases with activated CD4+ T cells (figure 6B,C). The mRNA levels of CD4 and ICOS were significantly increased after
MWA (figure 6B). Also, the PIK3R1 mRNA increased after MWA (figure 6B). Importantly, the mRNA levels of ICOS downstream factors, including JNK and ERK, but not p38, were significantly increased. Furthermore, the mRNA levels of IFN- $\gamma$ and the Th1 associated transcription factor T-bet were also significantly increased (figure 6B), while the CD8 mRNA level did not significantly increase. On the other hand, the ICOS pathway was not activated by surgery in breast cancer (online supplemental figure $3 \mathrm{~B}$ ).

\section{MWA-induced Th1-type immune response mainly in non- luminal breast cancers}

Non-luminal breast cancers (HER2+ breast cancer and TNBC) are more immunogenic than luminal breast cancers, ${ }^{3}{ }^{37}$ with more tumor-infiltrating lymphocytes in non-luminal breast cancers from analysis of biopsy samples in this study (detailed data not shown). The changes in MWA-induced immune response were examined in luminal-HER2-negative breast cancers (luminal group) and non-luminal breast cancers. In non-luminal breast cancers $(n=14)$, the peripheral percentages of $\mathrm{CD} 4+$ and ICOS+CD4+ T cells were significantly increased after MWA treatment (online supplemental figure 4A), but not CD8+ T cells. However, the peripheral percentages of all T cells, CD4+, ICOS+CD4+ and CD8+ T cells, were not significantly increased after MWA in luminal breast cancers $(n=19$, online supplemental figure $4 A)$.

The changes in Th1 cytokines were examined after MWA treatment. We found that the levels of IFN- $\gamma$ $(\mathrm{p}=0.041)$ and IL-2 $(\mathrm{p}=0.044)$ significantly increased after MWA in non-luminal breast cancers but not in luminal breast cancers (online supplemental figure 4B). No significant changes in other cytokines were observed in non-luminal or luminal breast cancers after MWA treatment. Additionally, the percentage of CD4+ $\mathrm{T}_{\mathrm{EM}}$ significantly increased with a mean level of $34.29 \%$ after MWA (online supplemental figure 4C, $\mathrm{p}=0.037$ ) in non-luminal breast cancers, but no significant difference was observed in luminal breast cancers (online supplemental figure $4 \mathrm{C})$.

\section{MWA-induced T-cell response after surgical resection}

Because MWA is not the standard local therapy for breast cancer currently, it is important to determine the MWAinduced immune response after surgical resection in the current situation. Of 20 patients who underwent MWA followed by prescheduled surgery 1 week later, the blood samples 4 weeks after MWA were available in 6 patients with enhanced CD4+ T cells 1 week after MWA. We found that the percentages of $\mathrm{T}$ cells and CD4+ T cells 4 weeks after MWA were still higher than those before MWA; however, the percentage of ICOS+ early-activated CD4+ T cells 4 weeks after MWA was significantly lower than that before MWA (figure 7A-C). Importantly, the percentage of CD8+ T cells significantly increased 4 weeks after MWA (figure 7D), but this difference was not observed 1 week after MWA. Moreover, the percentage of CD4+ $\mathrm{T}_{\mathrm{EM}} 4$ weeks after MWA was still significantly higher than that 
A

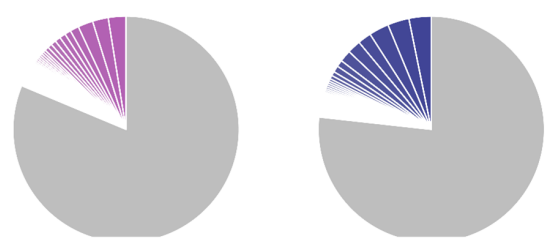

Case \#3

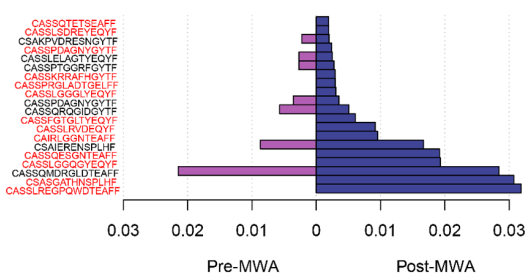

C
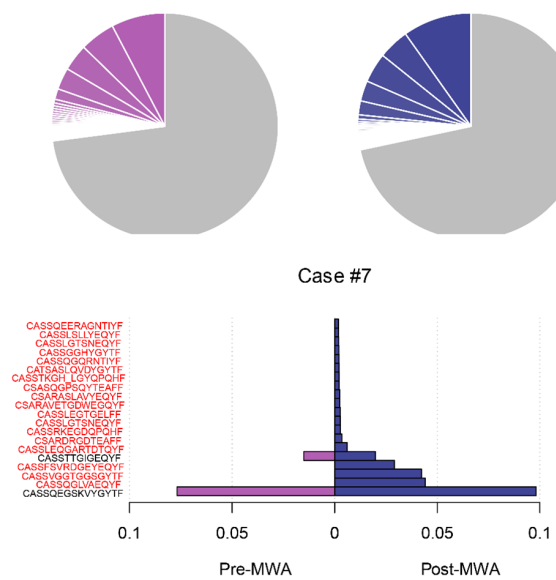

E

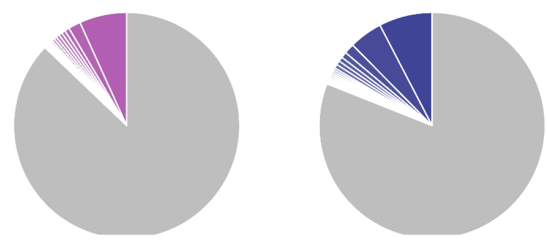

Case \#21

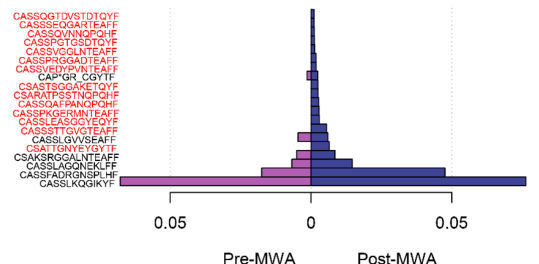

B

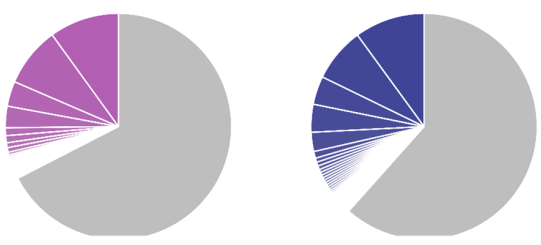

Case \#4

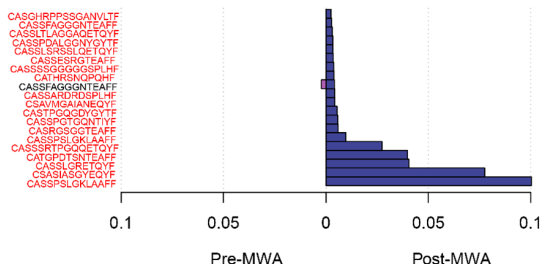

D

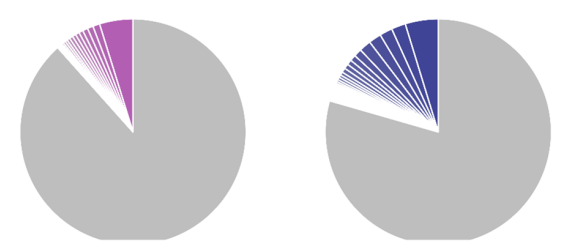

Case \#17

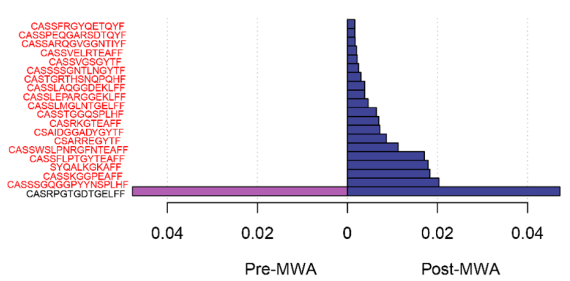

F

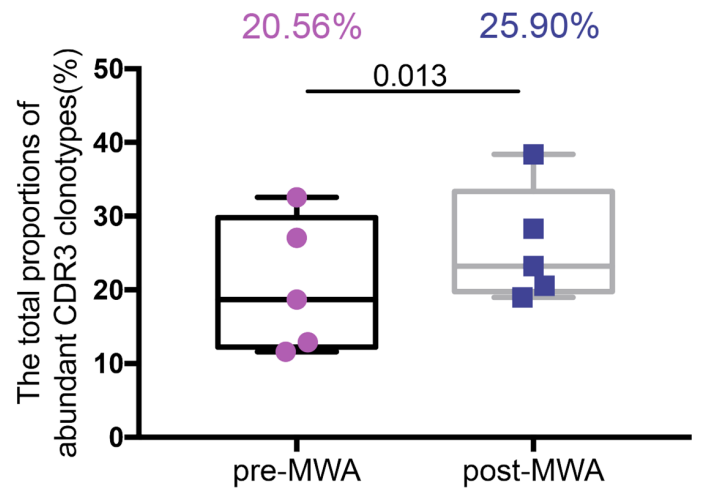

Figure 5 Expansion of TCR $\beta$ repertoire activated by MWA.(A-E) The distribution of the abundant TCR $\beta$ CDR3 clonotypes with a frequency of $0.1 \%$ or higher is presented in pie charts (top panel) (purple: pre-MWA, blue: post-MWA, gray: portion of CDR3 clonotypes below the frequency of $0.1 \%$ ). The top 20 abundant TCR $\beta$ CDR3 clonotypes newly appeared or increased after MWA (bottom panel) (purple bars: clonotypes that were rarely observed in pre-MWA samples, blue bars: top 20 abundant clonotypes observed in post-MWA samples; red: clonotypes that were completely absent in pre-MWA samples). Representative of five patients. (F) The total proportion of the abundant TCR $\beta$ CDR3 clonotypes with the frequency of $0.1 \%$ or higher was significantly increased after MWA ( $n=5)$. MWA, microwave ablation; TCR $\beta$, T-cell receptor $\beta$ 

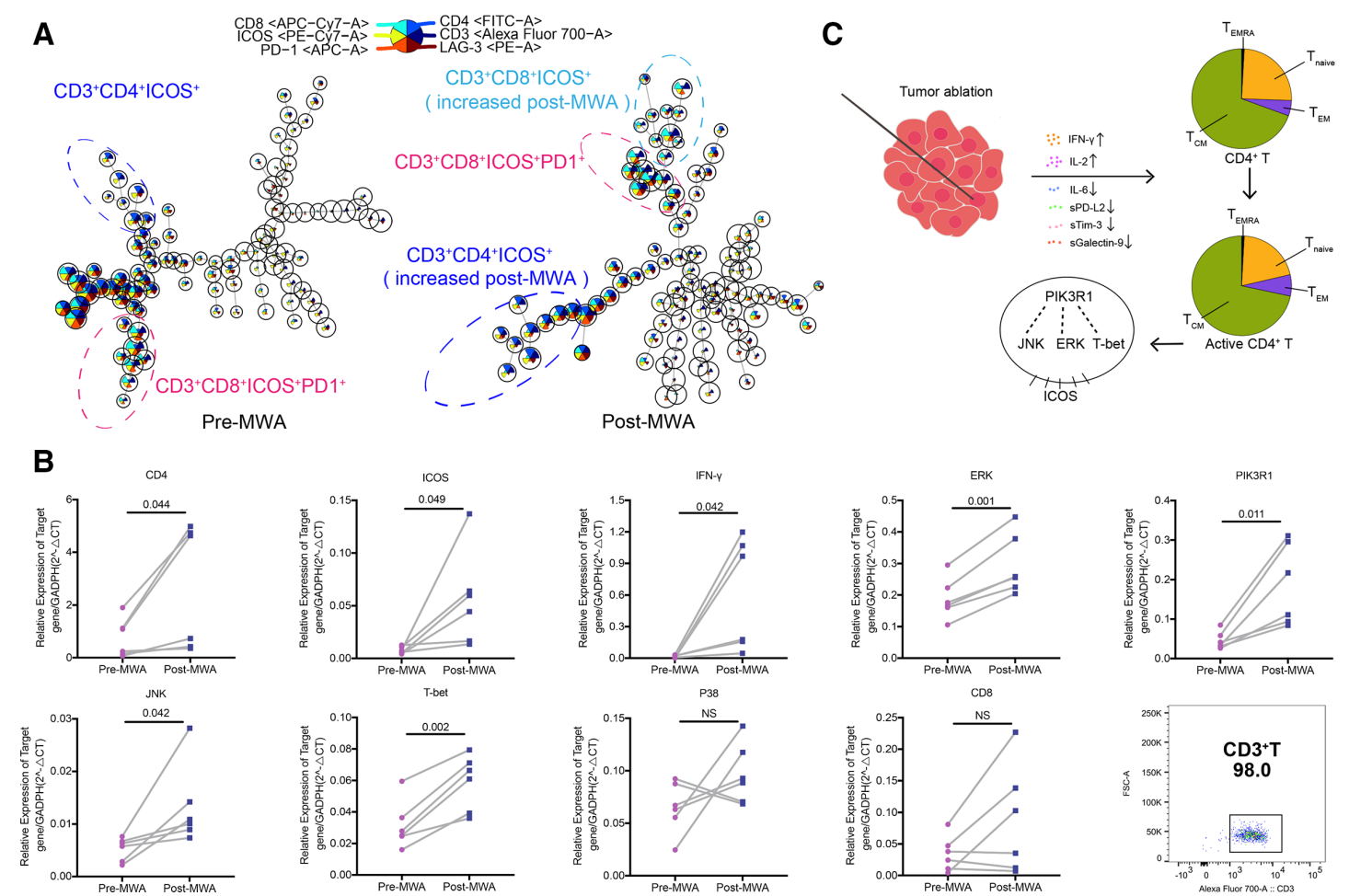

Figure 6 ICOS pathway of peripheral T cells activated by MWA of breast cancer. (A) Unsupervised clustering of T cells in patients with early-stage breast cancer pre-MWA and post-MWA. Large cell populations are highlighted. (B) The relative mRNA expressions of the ICOS pathway in peripheral T cells before and after MWA $(n=6)$. (C) MWA-induced Th1-type immune response via ICOS pathway. IFN- $\gamma$, interferon gamma; ICOS, inducible co-stimulator; IL, interleukin; MWA, microwave ablation; NS, not significant.

before MWA (figure 7E), with a lower level of naïve CD4+ T cells (figure 7F).

\section{DISCUSSION}

Despite great advances in breast cancer therapies, innovative approaches to breast cancer therapy are still needed to reduce relapse. As the standard local treatment, surgery can induce systemic inflammation and immunosuppression postoperatively. ${ }^{13} 14$ As a minimally invasive thermal therapy, MWA has been attempted in the treatment of breast cancer, ${ }^{24}{ }^{25}$ and we found MWA was a promising local therapy for breast cancer with a high complete ablation rate in this study. To the best of our knowledge, the immune response induced by ablation of breast cancer has not been clearly documented. In this study, MWAinduced Th1-type immune response was reported in women with early-stage breast cancer. Importantly, CD4+ $\mathrm{T}_{\mathrm{EM}}$ response was induced by MWA, possibly providing long-term protection against breast cancer. Furthermore, the ICOS pathway of peripheral $\mathrm{T}$ cells was activated by MWA of breast cancer. TCR sequencing revealed MWA of primary tumor activated peripheral $\mathrm{T}$ lymphocyte expansion and recognized some cancer-specific antigens. This study may offer a potentially effective alternative for surgery to treat early-stage breast cancer, although longterm local effect and systemic immune response should be determined in the future.
Minimally invasive thermal therapies have been applied to treat small breast cancers in several phase I/II trials with small sample sizes, ${ }^{152438}$ but the long-term outcomes of these therapies in the treatment of breast cancer have seldom been reported. ${ }^{27} 39$ Although surgery completely removes the tumors from the body, MWA destroys tumors in situ and creates an antigen source for antitumor immunity. ${ }^{16}$ Immune response induced by minimally invasive thermal therapies in breast cancer has only been reported in one clinical study with 12 patients. ${ }^{8}$ Th1-associated cytokine levels are stable over time without $>1$ fold change in the cryotherapy-only group including six patients, and markedly elevations in these cytokines were observed in six cases treated with cryotherapy and ipilimumab. Activated T-cell response was observed in the cryotherapy and ipilimumab groups, but not in the cryotherapy-only group. In this study, the IFN- $\gamma$ level was significantly increased about $50 \%$ in MWA group with $>1$ fold change in six cases, and the IL-2 level was increased about 1.5-fold after MWA, without significant difference from that in the surgery group. Moreover, ICOS+ activated CD4+ T-cell response was observed after MWA. Th1-type immune response was induced by MWA compared with surgery, and less inflammatory microenvironment was caused by MWA than surgery. This postablation microenvironment may provide protection against tumors, unlike the systemic inflammatory and immunosuppressive microenvironments induced by surgery. ${ }^{13}$ The variable immune 
A

B

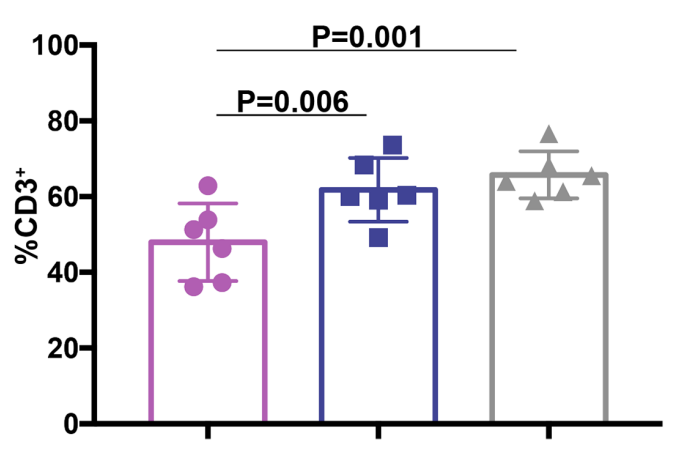

C

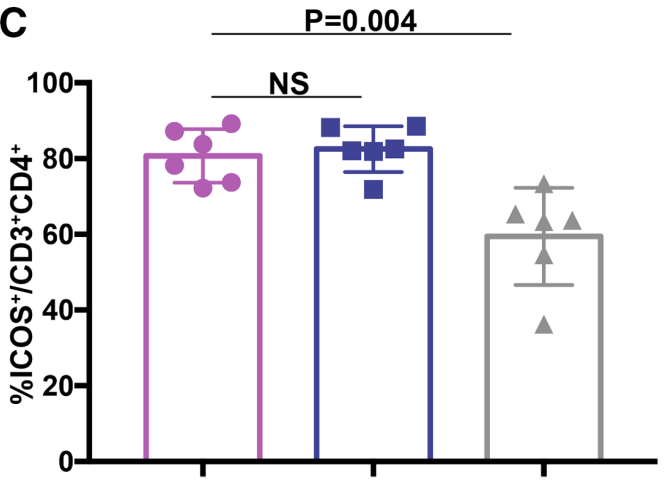

E

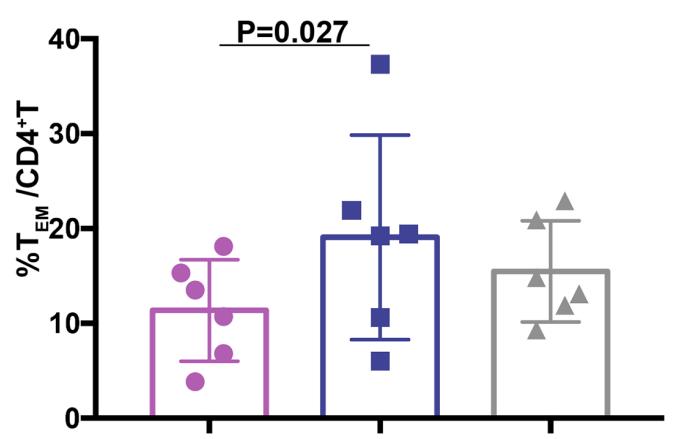

Pre-MWA — 1 week post MWA ^ 4 weeks post MWA

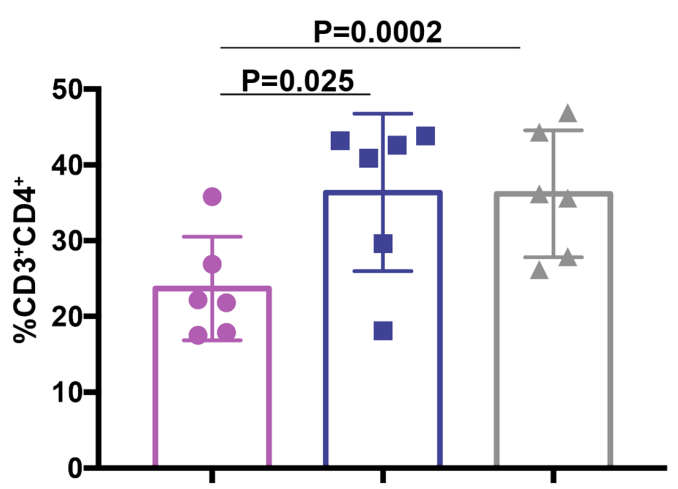

D

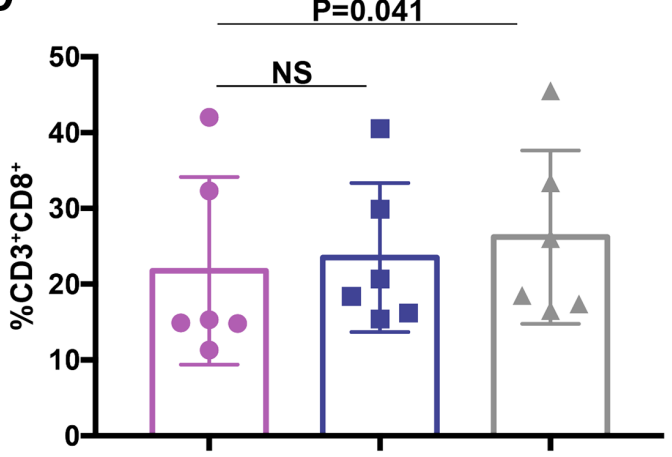

$\mathbf{F}$ $\mathrm{P}=\mathbf{0 . 0 4 9}$

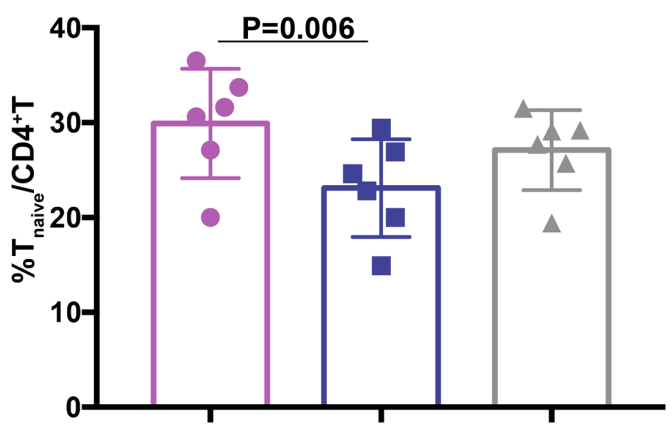

Figure 7 MWA-induced T-cell response after surgical resection. The percentages of peripheral T cells $(A), C D 4+(B)$, ICOS+CD4+ (C), CD8+ (D) and CD4+ effector memory $\left(T_{E M}\right)(E)$, and CD4+ naïve $(F)$ T cells 4 weeks after MWA treatment (followed by surgical resection 1 week later), 1 week after MWA and before MWA $(n=6)$. Data are presented as mean $\pm S D$. ICOS, inducible co-stimulator; MWA, microwave ablation; NS, not significant; $\mathrm{T}_{\mathrm{EM}}$, effector memory $\mathrm{T}$ cell; $\mathrm{T}_{\text {naive }}$, naïve $\mathrm{T}$ cell.

response to thermal ablation has been attributed to the balance between coagulative necrosis of the central ablation zone and apoptosis of periablational tissue. ${ }^{9}$ Necrosis results in an expulsion of intracellular contents, including DNA, RNA, HSPs and HMGB1, which alert the immune system; however, apoptosis may induce immunosuppression towards the antigens of the cell. High-power MWA leads to little local periablational inflammation with a very narrow rim of periablational tissue that is exposed to non-lethal levels of heating. ${ }^{40}$ Different from other minimally invasive therapies, MWA-induced immune response may mainly rely on the coagulative necrosis. MWA may be a promising approach in inducing immune changes for breast cancer, although the difference between MWA and other ablations including cryotherapy should be determined in the future.

Preclinical studies ${ }^{9} 2041$ have suggested weak Th1type immune response is induced by minimally invasive thermal therapies, and combined therapy can enhance Th1-type response and cytotoxic T-cell response. ${ }^{20} 4243$ However, the tumor cells used in preclinical studies have relatively high antigenicity, ${ }^{20}{ }^{41-43}$ such as $4 \mathrm{~T} 1$ breast cancer cell, BL6-10 melanoma cell, EG7 thymoma cell, and MB49 urothelial carcinoma cell. In this study with 
cold breast cancers, weak Th1-type immune response was found in patients with breast cancer treated with MWA in comparison to the systemic inflammation and immunosuppression microenvironments induced by standard surgery. Although the MWA-induced immune response was weak, activated CD8+ T-cell response was observed in several cases 1 week after MWA. Moreover, the percentage of cytotoxic CD8+ T cells significantly increased 4 weeks after MWA in selected cases. Therefore, it is important to investigate the group of patients who will present strong immune response induced by MWA. Since HER2+ breast cancer and TNBC are more immunogenic than luminal breast cancers, ${ }^{3} 37$ MWA-induced immune response between luminal and non-luminal breast cancers may be different. Interestingly, we found that the Th1-type immune response was mainly observed after MWA in non-luminal breast cancers. Our results also indicate that luminal-HER2-negative breast cancer is a really cold type of breast cancer, very different from non-luminal breast cancers. For relative 'hot' non-luminal breast cancer, not only immediately immune response was induced by MWA, but also effective secondary immunologic responses may arise. For luminal breast cancers, no significant immune response and systemic inflammation/immunosuppression were induced by MWA. Future studies are still needed to identify patients who will show strong MWAinduced immune responses.

ICOS, a member of the CD28 costimulatory family, is upregulated on the surface of $\mathrm{T}$ cells following T-cell activation. ${ }^{34}$ ICOS+CD4+ T cells produce greater levels of IFN- $\gamma$ compared with ICOS-CD4+ T cells, and ICOS+CD4+ $\mathrm{T}$ cells in the peripheral blood are related to increased survival after CTLA-4 blockade. In this study, we found that the ICOS pathway was activated and IFN- $\gamma$ increased by MWA but not surgery treatment of breast cancer. Previous studies ${ }^{45}$ have reported CTLA-4 blockade leads to a higher frequency of ICOS+CD4+ T cells, and providing additional signal to the ICOS pathway in the setting of CTLA-4 blockade markedly enhances the antitumor efficacy. MWA, which can provide additional signal to the ICOS pathway, combined with CTLA-4 blockade may be a promising strategy for the treatment of breast cancer. Moreover, we found that PD-1+exhausted CD8+ T-cell population was a major T-cell population in patients with breast cancer, which was not significantly influenced by MWA or surgery. PD-1 blockade may enhance the adaptive immune response induced by MWA in these patients.

Several potential limitations of our study should be considered. First, the previous study has found that peripheral T-cell response was induced by cryotherapy and ipilimumab 1 week after therapy, and the elevation from baseline subsequently declined. Furthermore, most patients underwent systemic therapy within 1 month postsurgery, by which the immune response may be influenced. Although significant immune response was observed 1 week after MWA in our present study, the long-term immune response and the effect of this response on clinical outcome are still unknown. Second, tumor infiltrated lymphocytes were not analyzed in this study because of too few viable cells obtained 1 week after MWA, and this local response may be determined for a long time interval after ablation in the future. Third, we cannot exclude the relationship between the immune response and the ablation of normal breast tissue absolutely, although little normal breast tissue surrounding the tumor was ablated. Moreover, the previous study ${ }^{46}$ has found that MWA of normal tissue has no effect on immune response in model mice. Fourth, since MWA is still not the standard local therapy for early-stage breast cancer and the MWA-induced immune response is weak, a certain combination strategy is urgently needed to test the local and systemic effects for the treatment of breast cancer.

\section{CONCLUSIONS}

As a minimally invasive therapy, MWA is an effective local treatment for breast cancer. MWA-induced Th1-type immune response and CD4+ $\mathrm{T}_{\mathrm{EM}}$ response were found. Considering surgery-induced systemic inflammation and immunosuppression microenvironments, MWA might be a trigger of antitumor immunity in breast cancer, especially for non-luminal breast cancer. Taken together, MWA may be a promising alternative to surgery for the local therapy of breast cancer, and combinatorial strategy using additional agents (eg, CTLA-4 inhibitor) which boost MWA-induced immune response could be considered as potential treatment for clinical study for early breast cancer therapy.

\section{Author affiliations}

${ }^{1}$ Department of Breast Surgery, The First Affiliated Hospital with Nanjing Medical University, Nanjing, China

${ }^{2}$ Jiangsu Key Lab of Cancer Biomarkers, Prevention and Treatment, Jiangsu Collaborative Innovation Center For Cancer Personalized Medicine, School of Public Health, Nanjing Medical University, Nanjing, China

${ }^{3}$ Department of Immunology, Nanjing Medical University, Nanjing, Jiangsu, China ${ }^{4}$ Department of Rheumatology and Immunology, The First Affiliated Hospital with Nanjing Medical University, Nanjing, China

${ }^{5}$ Pancreatic Center and Department of General Surgery, The First Affiliated Hospital with Nanjing Medical University, Nanjing, China

${ }^{6}$ Department of General Surgery, Huashan Hospital, Fudan University, Shanghai, China

${ }^{7}$ Department of Pathology, The First Affiliated Hospital with Nanjing Medical University, Nanjing, China

Contributors WZ, MY, WQ and HP contributed equally to this work. WZ and SW, as correspondence authors, contributed to the conception and design of the study, the analysis and interpretation of data, the revision of the article as well as final approval of the version to be submitted. WZ, MY, HP, WQ, HW, MQ, KZ, NC, XM, RW, $\mathrm{Li}$ Li and CW performed the experimental study and the statistical analysis, and drafted and revised the article. HX, LLing, YZ, CW, QD and XL participated in the clinical study, performed the statistical analysis, and drafted and revised the article. All authors read and approved the final version of the manuscript.

Funding This work was supported in part by the National Natural Science Foundation of China (81771953), the Six Kinds of Outstanding Talent Foundation of Jiangsu Province (WSW-014, to WZ), the Natural Science Foundation of Jiangsu Province (BK20180108), and a project funded by the Priority Academic Program Development of Jiangsu higher Education Institutions.

Competing interests None declared. 


\section{Patient consent for publication Not required.}

Ethics approval Microwave ablation has been approved for the treatment of solid tumors by the China Food and Drug Administration. The present study was approved by the institutional ethics committee of our hospital (2010-SR-003) and complied with the Declaration of Helsinki. Informed consent was obtained from all patients.

Provenance and peer review Not commissioned; externally peer reviewed.

Data availability statement All data relevant to the study are included in the article or uploaded as supplementary information. All data needed to evaluate the conclusions in this paper are present in the paper and/or the supplementary information file. Additional data related to this paper may be requested from the authors.

Supplemental material This content has been supplied by the author(s). It has not been vetted by BMJ Publishing Group Limited (BMJ) and may not have been peer-reviewed. Any opinions or recommendations discussed are solely those of the author(s) and are not endorsed by BMJ. BMJ disclaims all liability and responsibility arising from any reliance placed on the content. Where the content includes any translated material, BMJ does not warrant the accuracy and reliability of the translations (including but not limited to local regulations, clinical guidelines, terminology, drug names and drug dosages), and is not responsible for any error and/or omissions arising from translation and adaptation or otherwise.

Open access This is an open access article distributed in accordance with the Creative Commons Attribution Non Commercial (CC BY-NC 4.0) license, which permits others to distribute, remix, adapt, build upon this work non-commercially, and license their derivative works on different terms, provided the original work is properly cited, appropriate credit is given, any changes made indicated, and the use is non-commercial. See http://creativecommons.org/licenses/by-nc/4.0/.

\section{ORCID iD}

Wenbin Zhou http://orcid.org/0000-0003-1220-6797

\section{REFERENCES}

1 Siegel RL, Miller KD, Jemal A. Cancer statistics, 2019. CA Cancer J Clin 2019;69:7-34

2 Tolba MF, Omar HA. Immunotherapy, an evolving approach for the management of triple negative breast cancer: converting nonresponders to responders. Crit Rev Oncol Hematol 2018;122:202-7.

3 Emens LA. Breast cancer immunotherapy: facts and hopes. Clin Cancer Res 2018;24:511-20.

4 Robert C, Ribas A, Schachter J, et al. Pembrolizumab versus ipilimumab in advanced melanoma (KEYNOTE-006): post-hoc 5-year results from an open-label, multicentre, randomised, controlled, phase 3 study. Lancet Oncol 2019;20:1239-51.

5 Emens LA, Cruz C, Eder JP, et al. Long-Term clinical outcomes and biomarker analyses of Atezolizumab therapy for patients with metastatic triple-negative breast cancer: a phase 1 study. JAMA Oncol 2019;5:74-82.

6 Voorwerk L, Slagter M, Horlings HM, et al. Immune induction strategies in metastatic triple-negative breast cancer to enhance the sensitivity to PD-1 blockade: the tonic trial. Nat Med 2019;25:920-8.

7 Schumacher TN, Schreiber RD. Neoantigens in cancer immunotherapy. Science 2015;348:69-74.

8 McArthur HL, Diab A, Page DB, et al. A pilot study of preoperative single-dose ipilimumab and/or cryoablation in women with earlystage breast cancer with comprehensive immune profiling. Clin Cancer Res 2016;22:5729-37.

9 Chu KF, Dupuy DE. Thermal ablation of tumours: biological mechanisms and advances in therapy. Nat Rev Cancer 2014;14:199-208.

10 Zhao J, Wen X, Tian L, et al. Irreversible electroporation reverses resistance to immune checkpoint blockade in pancreatic cancer. Nat Commun 2019;10:899.

11 Liu Y, Dong Y, Kong L, et al. Abscopal effect of radiotherapy combined with immune checkpoint inhibitors. J Hematol Oncol 2018;11:104

12 Panigrahy D, Gartung A, Yang J, et al. Preoperative stimulation of resolution and inflammation blockade eradicates micrometastases. $J$ Clin Invest 2019;129:2964-79.

13 Coffey JC, Wang JH, Smith MJF, et al. Excisional surgery for cancer cure: therapy at a cost. Lancet Oncol 2003;4:760-8.

14 Decker D, Schondorf M, Bidlingmaier F, et al. Surgical stress induces a shift in the type-1/type-2 T-helper cell balance, suggesting down-regulation of cell-mediated and up-regulation of antibody-mediated immunity commensurate to the trauma. Surgery 1996;119:316-25.

15 Fornage BD, Hunt KK. Image-Guided percutaneous ablation of smal breast cancer: which technique is leading the pack? Technol Cancer Res Treat 2015;14:209-11.

16 den Brok MHMGM, Sutmuller RPM, van der Voort R, et al. In situ tumor ablation creates an antigen source for the generation of antitumor immunity. Cancer Res 2004;64:4024-9.

17 He K, Liu P, Xu LX. The cryo-thermal therapy eradicated melanoma in mice by eliciting $\mathrm{CD} 4^{+}$T-cell-mediated antitumor memory immune response. Cell Death Dis 2017:8:e2703.

18 Xu A, Zhang L, Yuan J. TLR9 agonist enhances radiofrequency ablation-induced CTL responses, leading to the potent inhibition of primary tumor growth and lung metastasis. Cell Mol Immunol 2018.

19 Behm B, Di Fazio P, Michl P, et al. Additive antitumour response to the rabbit VX2 hepatoma by combined radio frequency ablation and toll like receptor 9 stimulation. Gut 2016;65:134-43.

20 Li L, Wang W, Pan H, et al. Microwave ablation combined with OK432 induces Th1-type response and specific antitumor immunity in a murine model of breast cancer. J Transl Med 2017;15:23.

21 Takaki H, Imai N, Thomas CT, et al. Changes in peripheral blood Tcell balance after percutaneous tumor ablation. Minim Invasive Ther Allied Technol 2017;26:331-7.

22 Shi L, Chen L, Wu C, et al. PD-1 blockade boosts radiofrequency Ablation-Elicited adaptive immune responses against tumor. Clin Cancer Res 2016;22:1173-84.

23 Zerbini A, Pilli M, Laccabue D, et al. Radiofrequency thermal ablation for hepatocellular carcinoma stimulates autologous NK-cell response. Gastroenterology 2010;138:1931-42.

24 Zhou W, Zha X, Liu X, et al. US-guided percutaneous microwave coagulation of small breast cancers: a clinical study. Radiology 2012;263:364-73.

25 Zhou W, Jiang Y, Chen L, et al. Image and pathological changes after microwave ablation of breast cancer: a pilot study. Eur J Radiol 2014;83:1771-7.

26 Zhou W, Liang M, Pan $\mathrm{H}$, et al. Comparison of ablation zones among different tissues using 2450-MHz cooled-shaft microwave antenna: results in ex vivo porcine models. PLoS One 2013;8:e71873.

27 Palussière J, Henriques C, Mauriac L, et al. Radiofrequency ablation as a substitute for surgery in elderly patients with nonresected breast cancer: pilot study with long-term outcomes. Radiology 2012;264:597-605.

28 Frigola $\mathrm{X}$, Inman BA, Lohse CM, et al. Identification of a soluble form of $\mathrm{B} 7-\mathrm{H} 1$ that retains immunosuppressive activity and is associated with aggressive renal cell carcinoma. Clin Cancer Res 2011;17:1915-23.

29 Rossille D, Gressier M, Damotte D, et al. High level of soluble programmed cell death ligand 1 in blood impacts overall survival in aggressive diffuse large B-cell lymphoma: results from a French multicenter clinical trial. Leukemia 2014;28:2367-75.

30 Akutsu Y, Murakami K, Kano M, et al. The concentration of programmed cell death-ligand 1 in the peripheral blood is a useful biomarker for esophageal squamous cell carcinoma. Esophagus 2018;15:103-8.

31 Vahidi $\mathrm{Y}$, Faghih Z, Talei A-R, et al. Memory $\mathrm{CD} 4^{+} \mathrm{T}$ cell subsets in tumor draining lymph nodes of breast cancer patients: $A$ focus on $T$ stem cell memory cells. Cell Oncol 2018;41:1-11.

32 Sathaliyawala T, Kubota M, Yudanin N, et al. Distribution and compartmentalization of human circulating and tissue-resident memory T cell subsets. Immunity 2013;38:187-97.

33 Thome JJC, Bickham KL, Ohmura Y, et al. Early-Life compartmentalization of human $\mathrm{T}$ cell differentiation and regulatory function in mucosal and lymphoid tissues. Nat Med 2016;22:72-7.

34 Simpson TR, Quezada SA, Allison JP. Regulation of CD4 T cell activation and effector function by inducible costimulator (ICOS). Curr Opin Immunol 2010;22:326-32.

35 Grimbacher B, Hutloff A, Schlesier M, et al. Homozygous loss of ICOS is associated with adult-onset common variable immunodeficiency. Nat Immunol 2003;4:261-8.

36 Takahashi N, Matsumoto K, Saito H, et al. Impaired CD4 and CD8 effector function and decreased memory T cell populations in ICOSdeficient patients. J Immunol 2009;182:5515-27.

37 Denkert C, von Minckwitz G, Darb-Esfahani S, et al. TumourInfiltrating lymphocytes and prognosis in different subtypes of breast cancer: a pooled analysis of 3771 patients treated with neoadjuvant therapy. Lancet Oncol 2018;19:40-50.

38 Simmons RM, Ballman KV, Cox C, et al. A phase II trial exploring the success of cryoablation therapy in the treatment of invasive breast carcinoma: results from ACOSOG (Alliance) Z1072. Ann Surg Oncol 2016;23:2438-45. 
39 Cazzato RL, de Lara CT, Buy X, et al. Single-Centre experience with percutaneous cryoablation of breast cancer in 23 consecutive nonsurgical patients. Cardiovasc Intervent Radiol 2015;38:1237-43.

40 Velez E, Goldberg SN, Kumar G, et al. Hepatic thermal ablation: effect of device and heating parameters on local tissue reactions and distant tumor growth. Radiology 2016;281:782-92.

41 Dromi SA, Walsh MP, Herby S, et al. Radiofrequency ablation induces antigen-presenting cell infiltration and amplification of weak tumorinduced immunity. Radiology 2009;251:58-66.

42 Xue T, Liu P, Zhou Y, et al. Interleukin-6 Induced "Acute" Phenotypic Microenvironment Promotes Th1 Anti-Tumor Immunity in CryoThermal Therapy Revealed By Shotgun and Parallel Reaction Monitoring Proteomics. Theranostics 2016;6:773-94.
43 Xu A, Zhang L, Yuan J, et al. TLR9 agonist enhances radiofrequency ablation-induced CTL responses, leading to the potent inhibition of primary tumor growth and lung metastasis. Cell Mol Immunol 2019:16:820-32.

44 Carthon BC, Wolchok JD, Yuan J, et al. Preoperative CTLA-4 blockade: tolerability and immune monitoring in the setting of a presurgical clinical trial. Clin Cancer Res 2010;16:2861-71.

45 Fan X, Quezada SA, Sepulveda MA, et al. Engagement of the ICOS pathway markedly enhances efficacy of CTLA-4 blockade in cancer immunotherapy. J Exp Med 2014;211:715-25.

46 Yu M, Pan H, Che N, et al. Microwave ablation of primary breast cancer inhibits metastatic progression in model mice via activation of natural killer cells. Cell Mol Immunol 2020. doi:10.1038/s41423-0200449-0. [Epub ahead of print: 08 May 2020]. 